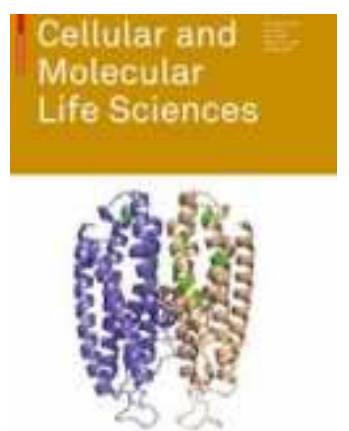

\title{
Characterisation of the self-palmitoylation activity of the transport protein particle component Bet3
}

\begin{tabular}{|r|l|}
\hline Journal: & Cellular and Molecular Life Sciences \\
\hline Manuscript ID: & CMLS-2010-0024.R1 \\
\hline Manuscript Type: & Research Article \\
\hline Key Words: & vesicular transport, tethering, TRAPP, Bet3, palmitoylation \\
\hline \multicolumn{2}{|l}{} \\
\hline
\end{tabular}

\section{s) scholaronE \\ Manuscript Central}




\title{
Characterisation of the self-palmitoylation activity of the transport protein particle component Bet3
}

\author{
Daniel Kümmel ${ }^{1,2^{*}}$, Julia Walter ${ }^{3 *}$, Martin Heck ${ }^{4}$, \\ Udo Heinemann ${ }^{1,2}$ and Michael Veit ${ }^{3 \#}$
}

\footnotetext{
${ }^{1}$ Max Delbrück Center for Molecular Medicine, Robert-Rössle-Str. 10, 13125 Berlin

${ }^{2}$ Institute for Chemistry and Biochemistry, Freie Universität, Takustr. 6, 14195 Berlin

${ }^{3}$ Department of Immunology and Molecular Biology, Freie Universität, Philippstr. 13, 10115 Berlin

${ }^{4}$ Institut für Medizinische Physik und Biophysik, Charité - Universitätsmedizin Berlin, Charitéplatz 1, 10117 Berlin, Germany
}

Running title: self-palmitoylation of Bet3

*: both authors contributed equally to this work

\#Address correspondence to Michael Veit, Ph.D., Immunology and Molecular Biology, Vet.-Med.

Faculty, Freie Universität, Philippstr. 13, 10115 Berlin, Germany. Fax: +493020936171, E-mail: mveit@zedat.fu-berlin.de. 


\begin{abstract}
Bet3, a transport protein particle component involved in vesicular trafficking, contains a hydrophobic tunnel occupied by a fatty acid linked to cysteine 68 . We reported that Bet3 has a unique selfpalmitoylating activity. Here we show that mutation of arginine 67 reduced self-palmitoylation of Bet3, but the effect was compensated by increasing the $\mathrm{pH}$. Thus, arginine helps to deprotonate cysteine such that it could function as nucleophile in the acylation reaction which is supported by the structural analysis of non-acylated Bet3. Using fluorescence spectroscopy we show that long-chain acyl-CoAs bind with micromolar affinity to Bet3, whereas shorter-chain acyl-CoAs do not interact. Mutants with a deleted acylation site or a blocked tunnel bind to Pal-CoA, only the latter with slightly reduced affinity. Bet3 contains three binding sites for Pal-CoA, but their number was reduced to two in the mutant with an obstructed tunnel indicating that Bet 3 contains binding sites on its surface.
\end{abstract}

Key words: Golgi, vesicular transport, tethering, TRAPP, Bet3, palmitoylation, acyl-CoA 


\section{Introduction}

Bet3 was identified to be a component of the multi-subunit TRAPP (transport protein particle) trafficking complex that plays an important role for the recruitment of ER-derived vesicles to the Golgi apparatus [1-5]. Two TRAPP complexes have been characterised that differ in size, localization within the Golgi and function [2]. Both TRAPP complexes facilitate nucleotide exchange on the small GTPase Ypt1 [6-8]. X-ray crystallography with human and mouse Bet3 revealed a novel protein fold and, surprisingly, a long chain fatty acid covalently bound via a thioester linkage to the conserved residue cysteine 68 . The carbon chain is not exposed at the surface of the molecule, but completely buried inside a hydrophobic tunnel $[9,10]$. Acylated Bet3 forms homodimers and also heterodimers with structurally related, but non-acylated TRAPP subunits, such as TPC6/Trs33 [11-14].

A variety of fatty acids may be attached to cysteine residues of proteins through an acylation reaction. S-acylation (also termed palmitoylation) may be dynamic with rapid cycles of acylation and deacylation, in contrast to other hydrophobic modifications, i.e. myristoylation or isoprenylation. Palmitoylation controls targeting of the modified protein to membranes or membrane subdomains, affects protein-protein interactions and influences the stability of proteins [15]. Palmitoylation of proteins is also involved in intracellular transport. Pal-CoA, the lipid donor in the palmitoylation reaction, stimulates transport through the Golgi in vitro, supporting budding as well as fusion of vesicles. It was suggested that this stimulatory effect might be due to palmitoylation of a protein, which thereby gets activated to function in the transport reaction [16, 17].

Palmitoyl-CoA is capable of spontaneously S-acylating cysteine residues of several proteins and especially of peptides at their natural palmitoylation sites in vitro [18-25]. Most of these reactions are unlikely to operate inside cells. They are either too slow or require unphysiologically high pH values or Pal-CoA concentrations to account for palmitoylation in vivo.

The family of DHHC-CRD proteins (polytopic membrane proteins with the sequence DHHC and a cysteine-rich domain) has been identified as acyltransferases for a variety of substrate proteins in yeast and mammals [26]. However, Bet3 is unique in this respect since it has strong self-palmitoylating activity also in vivo. Incubation of purified Bet 3 with $\left[{ }^{3} \mathrm{H}\right]$-palmitoyl-CoA leads to a rapid and complete attachment of fatty acids to cysteine 68 [27]. This conclusion was supported by a study on the palmitoyl proteome of $S$. cerevisiae. Various DHHC proteins were deleted, and the effect on palmitoylation of individual proteins was determined. Only Bet3 showed a palmitoylation that was undiminished in any of the DHHC-mutant strains strongly arguing in favour of an autocatalytic mode for its acylation [28].

The only mechanistic understanding of acylation reactions of proteins has been derived from structural studies on the N-myristoyl-transferase (NMT) which attaches the rare C:14 carbon chain via an amide linkage to an N-terminal glycine residue. NMT binds almost exclusively myristoyl-CoA with nanomolar affinity, the binding site contains all the elements responsible for acyl chain-length measurement. Acyl transfer occurs through the nucleophilic addition-elimination reaction: an 
oxyanion hole formed by main-chain atoms of NMT polarizes the thioester carbonyl of Myr-CoA and stabilizes the transition state while deprotonation of the ammonium of the Gly acceptor is mediated by NMT's C-terminal carboxylate [29].

Here we have characterised the self-palmitoylation reaction of Bet3 in more detail. We found that Bet3 has an inherent propensity to be autoacylated which is fine-tuned by amino acids located in the vicinity of the acylation site. Especially the conserved arginine 67 enhances the acylation reaction, probably by facilitating deprotonation of cysteine 68 such that it could function as a nucleophile attacking the carbonyl of the C-S bond in Pal-CoA. Acyl-CoAs containing long chain fatty acids bind with micromolar affinity to Bet3, whereas shorter-chain acyl-CoAs do not interact. Bet3 mutants with a blocked tunnel bind to Pal-CoA with only slightly reduced affinity and revealed two acyl-CoA binding sites instead of three calculated for wild-type Bet3. We conclude that Bet 3 contains acyl-CoA binding sites on its surface which might be required to extract Pal-CoA from intracellular membranes. Our findings might be helpful to understand palmitoylation performed by DHHC proteins which are promising, but poorly characterised drug targets [26]. 


\section{Materials and Methods}

\section{Recombinant protein expression}

For expression of GST fusion protein in E. coli, human Bet3 cDNA was cloned into the bacterial expression vector pGEX-4T1 (GE Healthcare, Munich, Germany). Site-directed mutagenesis was performed by overlap extension PCR [30]. E. coli BL21 (DE3) cells were transformed with the resulting plasmids. An overnight culture was inoculated in YT medium containing $100 \mu \mathrm{g} / \mathrm{ml}$ ampicillin. The preculture was diluted 20 -fold in the same medium, grown to $\mathrm{OD}_{600} 0.7$ at $37^{\circ} \mathrm{C}$, and induced with $1 \mu \mathrm{M}$ isopropyl- $\beta$-D-thiogalactopyranoside. Growth was continued for $3 \mathrm{~h}$, cells were harvested by centrifugation, and pellets were stored at $-80{ }^{\circ} \mathrm{C}$. To obtain GST-Bet 3 cells were lysed using a French press (SLM-AMINCO) in PBS (phosphate buffered saline) supplemented with $1 \mathrm{mM}$ PMSF, 1 mM DTT and DNAse I (Roche). A cleared lysate obtained by centrifugation was loaded on 2 ml glutathione-Sepharose (Amersham) in a polypropylene column. The column was washed with PBS, protein was eluted with elution buffer (50 mM Tris $\mathrm{pH} 8,10 \mathrm{mM} \mathrm{GSH}$ ), and elution fractions were dialyzed against $25 \mathrm{mM}$ Tris, $\mathrm{pH} 8.4,120 \mathrm{mM} \mathrm{NaCl}$.

To obtain Bet3 without GST-tag the following procedure was used: In some experiments cells expressing GST-Bet3 were lysed by sonication in PBS supplemented with $1 \mathrm{mM}$ PMSF, $1 \mathrm{mM}$ DTT, and DNase I (Boehringer, Mannheim, Germany). A cleared lysate obtained by centrifugation was loaded on $5 \mathrm{ml}$ of glutathione-Sepharose (GE Healthcare) in a polypropylene column. The column was washed with PBS. GST was cleaved by on-column incubation with $100 \mathrm{U}$ thrombin over night at room temperature. Protein was eluted with elution buffer $(50 \mathrm{mM}$ Tris, $1 \mathrm{M} \mathrm{NaCl}, \mathrm{pH} 7.4)$ over a $1 \mathrm{ml}$ HiTrap benzamidine column (GE Healthcare), to retain thrombin. Protein solutions were concentrated and the buffer exchanged to PBS with Amicon Ultra Centrifugal Filter devices (10,000 MWCO) (Millipore).

\section{Palmitoylation assay}

$\left[{ }^{3} \mathrm{H}\right]-\mathrm{Pal}-\mathrm{CoA}$, prepared as described [22], was dried in a SpeedVac and dissolved in $20 \mathrm{mM}$ Tris-HCl (pH 7.4), $120 \mathrm{mM} \mathrm{NaCl}, 0.05 \%$ Triton X-100 to yield a final concentration of $100,000 \mathrm{CPM} / \mu$. The palmitoylation reaction included purified protein, GST-Bet3 wild type and mutants, $(0.5-8 \mu \mathrm{l}, 10 \mu \mathrm{g}$, $4 \mu \mathrm{M}), 5 \mu \mathrm{l}$ of $\left[{ }^{3} \mathrm{H}\right]-\mathrm{Pal}-\mathrm{CoA}(1 \mu \mathrm{M}, 500,000 \mathrm{CPM})$, and $1 \mathrm{mM}$ DTT in a final volume of $100 \mu \mathrm{l}$ of PBS buffer and was incubated for $20 \mathrm{~min}$ at $30{ }^{\circ} \mathrm{C}$. One $\mathrm{ml}$ of chloroform/methanol (1:1) was added to the samples, and precipitated proteins were pelleted $(15 \mathrm{~min}, 14,000 \times \mathrm{g})$, washed, resuspended in 20 $\mu 1$ of non-reducing SDS-PAGE sample buffer, and boiled for $5 \mathrm{~min}$. Samples were subjected to SDSPAGE, staining with Coomassie, and fluorography for $5-48 \mathrm{~h}$. The palmitoylation assays shown in figures 1-3 and 5-7 were performed at least two times with similar results.

\section{Structure determination}


The Trs33B:Bet3C68A complex was purified essentially as described before [13]. The protein was concentrated to $12 \mathrm{mg} / \mathrm{ml}$ in buffer containing $20 \mathrm{mM}$ Tris, $200 \mathrm{mM} \mathrm{NaCl}, 2 \mathrm{mM}$ DTT, pH 7.5 and 1 $\mathrm{mM}$ Myr-X-CoA, a non-hydrolysable myristoyl-CoA thioether. Crystals were obtained at $20^{\circ} \mathrm{C}$ by the sitting-drop method using a semi-automated dispensing system [31] in conditions containing $22 \%$ PEG3350, 0.2 $\mathrm{M} \mathrm{Li}_{2} \mathrm{SO}_{4}, 0.1 \mathrm{M}$ Bis-Tris pH 6.5. Crystals were flash-frozen in liquid nitrogen in cryo solution containing 20\% PEG3350, 15\% glycerol, 0.2 $\mathrm{M} \mathrm{Li}_{2} \mathrm{SO}_{4}, 0.1 \mathrm{M}$ BisTris pH 5.6, $250 \mu \mathrm{M}$ Myr$\mathrm{X}-\mathrm{CoA}$, and data were collected at ESRF BM14. Initial phases were obtained using Phaser [32] with the Trs33B:Bet3 complex (PDB: 2CFH) as a search model. After model bias removal (http://tuna.tamu.edu/), the final structure was obtained in several rounds of model building with Coot [33] and refinement using REFMAC5 [34]. The coordinates and diffraction amplitudes were deposited in the Protein Data Bank with accession code $3 \mathrm{KXC}$.

\section{Fluorescence spectroscopy}

Purified Bet 3 was diluted with Tris buffer ( $5 \mathrm{mM}$ Tris, $100 \mathrm{mM} \mathrm{NaCl}, \mathrm{pH}$ 7.4) to a concentration of 10 $\mu \mathrm{M}$. Measurements were carried out with a Cary Eclipse Fluorescence spectrophotometer (Varian) at room temperature using a quartz glass cuvette (100 $\mu$ l volume). Samples were excited at $295 \mathrm{~nm}$, and the emission scanned in the range of 310-450 nm. Each scan was repeated five times, and scanned graphs were averaged. Emission maxima were read out at $344 \mathrm{~nm}$. Emission of the protein was recorded and then the acyl-CoA concentration increased stepwise by adding $1 \mu$ lof stock solution (in Tris buffer, concentration $1 \mathrm{mM}$ to $100 \mathrm{mM}$ ). The reduction of the fluorescence intensity was normalized $\left(\mathrm{F}_{0}-\mathrm{F} / \mathrm{F}_{0} ; \mathrm{F}_{0}\right.$ : fluorescence intensity without ligand, $\mathrm{F}$ : fluorescence intensity at a certain acyl-CoA concentration) and plotted against the acyl-CoA concentration. Taking substrate depletion into account, the following equation can be derived for acyl-CoA binding to Bet3 [35]:

$$
Y=\frac{K_{D}+L+n P-\sqrt{\left(K_{D}+L+n P\right)^{2}-(4 L n P)}}{2 n P / Y_{\max }}
$$

where $\mathrm{K}_{\mathrm{D}}$ denotes the apparent dissociation constant for acyl-CoA, $\mathrm{L}$ and $\mathrm{P}$ denote the ligand and protein concentration, respectively, $\mathrm{n}$ is the number of ligand binding sites per protein, and $\mathrm{Y}_{\max }$ is the final level of the relative fluorescence change. In all fits using Prism (GraphPad), protein concentration was fixed to $10 \mu \mathrm{M}$ and $\mathrm{K}_{\mathrm{D}}, \mathrm{n}$ and $\mathrm{Y}_{\max }$ were allowed to vary. The results shown were calculated from at least three different experiments.

\section{Circular dichroism}

For circular dichroism spectroscopy, protein samples of human Bet3 purified from E. coli were diluted to $0.16 \mathrm{mg} / \mathrm{ml}$ in PBS buffer. Measurements were carried out with a Jasco J720 spectropolarimeter at $20{ }^{\circ} \mathrm{C}$ from $200 \mathrm{~nm}$ to $260 \mathrm{~nm}$ with a $0.2 \mathrm{~cm}$ path-length cuvette. 


\section{Results}

\section{Characterisation of the mechanism of the Bet3 self-palmitoylation reaction}

Insertion of the fatty acid of Pal-CoA into the hydrophobic tunnel, leaving the CoA moiety outside, would be a plausible mechanism to initiate self-palmitoylation of Bet3. This would position the thioester bond of the Pal-CoA molecule at the entrance of the tunnel where the fatty acid acceptor, cysteine 68, is located. Acyl-chain transfer then might occur through nucleophilic attack of the sulphur of cysteine 68 on the carbonyl-C of the C-S bond in Pal-CoA to catalyse the generation of acylated Bet3. It has been postulated that, besides availability of an activated fatty acid (acyl-CoA), the formation of a deprotonated cysteine (a thiolate) controls S-acylation [36]. Free cysteine has a pKa value of 8.5 so that deprotonation occurs infrequently at the neutral $\mathrm{pH}$ of the cell. However, the apparent pKa values may differ widely for individual cysteines present inside a peptide chain because the local environment strongly influences deprotonation of the SH group [37].

We first analysed the effect of thiolates on self-palmitoylation of Bet3 by facilitating their formation using buffers with decreasing proton concentration. We compared wild-type GST-Bet3 and mutants with modifications of the acylation site. Cysteine 68 was changed to alanine (C68A), or to serine (C68S) which, due to its hydroxyl group retains a potential acylation site. The palmitoylation assays were performed with GST-Bet3 fusion proteins, since the GST tag provided stability and protection against proteolytical degradation. However, performimg the assays with Bet3 alone produced identical results (not shown). Another mutant contained glutamic acid instead of the strictly conserved arginine in the vicinity of the acylation site (R67E). Figure 1A depicts the structure of Bet 3 with the location of mutated amino acids highlighted.

Purified proteins were incubated with $\left[{ }^{3} \mathrm{H}\right]-\mathrm{Pal}-\mathrm{CoA}$ in buffer adjusted to $\mathrm{pH} 6.5,7.5$ or 8.5 for 20 min, and samples were subjected to SDS-PAGE and fluorography. At pH 6.5 only palmitoylation of wildtype Bet3 was readily detectable. The R67E mutant showed reduced acylation at neutral pH, probably by affecting deprotonation of cysteine 68. With increasing $\mathrm{pH}$ acylation of the mutants C68S and R67E becomes obvious, and at $\mathrm{pH} 8.5$ it is almost as strong as the acylation of Bet $3 \mathrm{wt}$ (Fig. 1B). Even faint acylation of $\mathrm{C68 \textrm {A }}$ becomes apparent at $\mathrm{pH} 8.5$, probably representing background acylation occuring at other cysteine residues. Two additional cysteines are found in human Bet3, residue 31, which is partially surface-exposed, and residue 132 , which is in the center of the protein so that unfolding at unphysiological $\mathrm{pH}$ would be a prerequisite for its palmitoylation.

Deprotonation of Cys 68 might be facilitated when amino acids in its vicinity serve as acceptor of the proton. Such a function has been proposed for the carboxylate present at the C-terminus of the $\mathrm{N}$ myristoyl transferase which accepts a proton from the designated acylation site in the protein, in this case the ammonium of an N-terminal glycine residue [29]. Likely candidates for a role as proton acceptors apparent from the structure of Bet3 are His 69, and the acidic amino acids Asp 70 and Glu 73 (Fig. 1A). In contrast to His 69, which is not present in yeast Bet3, Glu 73 and especially Asp 70 are partially conserved within Bet 3 orthologs and were therefore mutated to their respective amides. 
However, these Bet3 mutants were acylated at neutral pH to a similar (E73Q), or even slightly higher (D70N) extent as compared to Bet3 wt (Fig. 1C).

In the next set of experiments the self-palmitoylation activity of each Bet3 mutant was compared at various $\mathrm{pH}$ values between 5.5 and 9.5 (at more basic values the self-palmitoylation activity of Bet 3 is inhibited [27]). Palmitoylation of wild-type Bet3 increased gradually up to $\mathrm{pH} 8.5$, where it reached a plateau, indicating an apparent $\mathrm{pKa}$ of the reaction in the neutral range. The $\mathrm{pH}$ profiles of Bet3 mutants R67E, C68S and C68A are shifted toward basic $\mathrm{pH}$, which is most evident for the C68A mutant (Fig. 2A). In contrast, the $\mathrm{pH}$ profiles of D70N and E73Q mutants are shifted toward acidic $\mathrm{pH}$ (Fig. 2B), an observation that was even more striking when both amino acids were exchanged simultaneously (D70N-E73Q). The results suggest a specific influence of the mutations on the apparent $\mathrm{pKa}$ value of the palmitoylation reaction.

Self-palmitoylation of Bet3 proceeds rapidly, i.e. incorporation of $\left[{ }^{3} \mathrm{H}\right]$-palmitate saturates in less than ten minutes (Fig. 3 and [27]). In contrast, acylation of Bet3 mutants R67E, C68S and A82L, a mutant with an obstructed hydrophobic tunnel, advances much more slowly (Fig. 3), which suggests a reduced enzymatic activity.

Structure of the Trs33B:Bet3-C68A dimer crystallised in the presence of a non-hydrolysable acyl-CoA analogue

To provide a structural basis for the mechanism of Bet3 acylation we attempted the co-crystallization of the mutant in complex with non-hydrolysable myristoyl-CoA thioether (Myr-X-CoA) which binds to Bet3 as described below (see Fig. 5C). This protein-ligand complex should represent a reaction intermediate of Bet3 acylation. We determined the structure at 2-Á resolution using molecular replacement with the Trs33B:Bet3 complex (Table 1). To reduce model bias, the palmitoyl chain occupying the hydrophobic tunnel and the $\alpha 2-\alpha 3$ loop containing the palmitoylation site were excluded from the initial refinement, and the model was submitted to the model bias removal server (http://tuna.tamu.edu/). Suprisingly, Myr-X-CoA was not present in the crystal, which was confirmed by generating an anomalous difference map from a data set collected at low energy (1.7 $\AA$ ): no sulphur signal from the ligand could be detected in the map (not shown). Nevertheless, the hydrophobic tunnel of Bet3 is occupied by an elongated density, which we found to be best accounted for by modelling a palmitate molecule (Fig. 4A). The palmitate was probably co-purified during the isolation of the protein from E. coli to stabilize the molecule [27]. Myr-X-CoA binds to Bet3 with higher affinity than palmitate (see Fig. 5 and 7, below), but the additional CoA moiety might have interfered with the formation of crystal contacts, which may have led to the formation of crystals containing palmitate instead. Although we did not achieve our goal to crystallize a Bet3-ligand complex, the first structure of the Bet3 C68A mutant has peculiar properties that might be interesting with regard to the mechanism of the acylation reaction. 


\section{Characterisation of the substrate requirements of the palmitoylation reaction}

Next we analysed the chemical composition required for a lipid molecule to function as ligand of Bet3. Increasing concentrations of unlabeled acyl-CoAs were added to the in vitro palmitoylation reaction. Myr- (C 14:0), Pal- (C 16:0), Stear- (C 18:0) and Oleoyl-CoA (C 18:1) almost completely inhibited $\left[{ }^{3} \mathrm{H}\right]$-palmitoylation of Bet3 at concentrations of around $10 \mu \mathrm{M}$ (Fig. 5a) indicating that they compete with $\left[{ }^{3} \mathrm{H}\right]-\mathrm{Pal}-\mathrm{CoA}$ for a binding site. In contrast, only a slight inhibition of $\left[{ }^{3} \mathrm{H}\right]-$ palmitoylation of Bet3 was observed when 1000-fold higher concentrations of acetyl- or octanoylCoA were used (Fig. 5B). It cannot be inferred from these experiments that all long chain acyl-CoAs are actually transferred to Bet3. Since myristate was found as the prevailing fatty acid covalently linked to Bet3 purified from E. coli [9], it is likely that the other acyl-CoAs can also be utilized by Bet3. But their ability to react with Bet3 is not a prerequisite for binding of acyl-CoAs to Bet3: The Myr-CoA analogue Myr-X-CoA inhibited self-palmitoylation of Bet3 at only marginally diminished concentration compared to Myr-CoA (Fig. 5C).

For a quantitative assessment of Bet3 palmitoylation, we employed fluorescence spectroscopy to analyse binding of the substrate to the protein. Bet 3 contains only one tryptophan residue at position 96, which is exposed at the surface of the molecule near the end of the hydrophobic tunnel (Fig. 1A). When excited at $295 \mathrm{~nm}$, purified Bet3 (without GST) displayed a broad emission peak between 330 and $360 \mathrm{~nm}$ (uppermost trace in Fig. 6a). Adding increasing amounts of CoAs with long chain fatty acids, i.e. Myr-CoA, Pal-CoA, Stear-CoA and Oleoyl-CoA to Bet3 quenched its intrinsic fluorescence gradually and yielded complete quenching at a final concentration in the lower micromolar range (shown exemplarily for Pal-CoA in Fig. 6A). In contrast, CoAs esterified to short carbon chains, i.e. acetyl-CoA (C 2:0, Fig. 6B) and octanoyl-CoA (C 8:0, not shown), did not completely quench the intrinsic fluorescence of Bet3, even at a final concentration of $10 \mathrm{mM}$. The reduction in fluorescence intensity was normalized and plotted against the acyl-CoA concentration (shown for palmitoyl- and acetyl- CoA in Fig. 6A-B, right panel). The data were fitted as described in "materials and methods" with an equation taking into account ligand depletion upon substrate binding. From the fitting, the number of acyl-CoA binding sites per protein and a value for the apparent dissociation constant for 
acyl-CoA $\left(K_{D}\right)$ (comprised of the individual $K_{D} S$ of each acyl-CoA binding site) were obtained (Table 2 and 3$)$.

When analysing the most common acyl-CoAs containing long chain, saturated fatty acids, i.e. MyrCoA, Pal-CoA and Stear-CoA, the intrinsic fluorescence of Bet3 was almost completely quenched at concentrations of around $50 \mu \mathrm{M} . \mathrm{K}_{\mathrm{D}}$ values of $2.0 \mu \mathrm{M}$ (Myr-CoA), $1.8 \mu \mathrm{M}$ (Pal-CoA) and $1.3 \mu \mathrm{M}$ (Stear-CoA) were calculated indicating that they bound with similar affinity to Bet3 (see Table 2). Almost identical results $\left(\mathrm{K}_{\mathrm{D}}: 2.1 \mu \mathrm{M}\right)$ were observed for an unsaturated acyl-CoA (oleoyl-CoA) indicating that a kink in the fatty acid does not diminish binding to Bet3. In contrast, $\mathrm{K}_{\mathrm{D}}$ values in the millimolar range were calculated for CoAs containing short acyl chains. (Fig. 6B and Table 2).

Does self-acylation of Bet 3 require activation of the fatty acid substrate? Addition of $\left[{ }^{3} \mathrm{H}\right]$-palmitate did not label Bet3, even when 10-fold higher radioactivity compared to $\left[{ }^{3} \mathrm{H}\right]$-Pal-CoA was used in the assay and when CoA and ATP were also present to possibly activate the fatty acid (data not shown). Free palmitate inhibited the palmitoylation reaction only at millimolar concentrations (Fig. 7A, left part). The other constituent of activated fatty acids, CoA, did not inhibit the self-palmitoylating activity of Bet 3 either, even at concentrations of $10 \mathrm{mM}$ (data not shown).

Thus, the requirements for a substrate to react with Bet3 are a long chain fatty acid linked to CoA, neither the carbon chain nor CoA alone interacts with micromolar affinity. We also asked whether other fatty acid-containing compounds, especially those used as inhibitors of protein palmitoylation, interact with Bet3. Lyso-phosphatidylcholine and tunicamycin both contain a long acyl chain, either bound to glycerol or (via sugar moieties) to adenine, and both inhibit self-palmitoylation of Bet3, with a half maximal inhibition at a concentration of around $100 \mu \mathrm{M}$ (Fig. 7B). In contrast, cerulenin, which contains an unsaturated, C8 carbon chain, does not inhibit the reaction, even at millimolar concentration. Cerulenin and tunicamycin are established inhibitors of fatty acid synthesis and Nlinked glycosylation, respectively, but have also been used by some as inhibitors of protein palmitoylation [38]. The presently most popular inhibitor of protein palmitoylation is 2-bromopalmitate [38], which also very efficiently blocks palmitoylation of Bet3 at low $\mu \mathrm{M}$ concentration (Fig. 7A) and binds to wild-type Bet3 with a $\mathrm{K}_{\mathrm{D}}$ value of $1.3 \mu \mathrm{M}$ (Table 2).

The palmitoylation reaction catalyzed by the yeast DHHC protein Akrlp is stimulated by ATP [39]. Surprisingly, however, ATP at concentrations of $10 \mathrm{mM}$ completely inhibited self-palmitoylation of Bet3. The phenomenon was specific for the adenine trinucleotide and required a hydrolysable ATP, since GTP and ATP $\gamma$ s did not block the reaction (Fig. 7C).

Determination of the number of Pal-CoA interaction sites in Bet3 and molecular requirements for binding

We next investigated whether Bet3 mutants with blocked or diminished self-palmitoylation activity have also a reduced binding affinity for the substrate. First we asked whether covalent transfer of the fatty acids to Cys 68 is a prerequisite for binding of acyl-CoAs to Bet3. In the fluorescence 
spectroscopy assay, Bet3 C68A revealed very similar $\mathrm{K}_{\mathrm{D}}$ values for binding of various acyl-CoAs compared to wild-type Bet3 (Fig. 6C and Table 2). Furthermore, binding of the other long chain acylCoAs was not altered either, and short chain acyl-CoAs did not bind (Table 2). Thus, wild-type Bet3 and Bet3 C68A have identical affinities for the lipid substrate, indicating that binding and fatty acid transfer are distinct events.

Next we tested our initial assumption that insertion of the fatty acid moiety into the hydrophobic tunnel is a prerequisite for binding of Pal-CoA to Bet3. Surprisingly, measuring the affinity for PalCoA of the Bet 3 mutant containing a narrowed tunnel (A82V) revealed a $K_{D}$ value of $4.0 \mu \mathrm{M}$, close to that of wild-type Bet3 (Fig. 6C and Table 3).

Since Bet3 A82V or A82L still shows residual self-palmitoylating activity at neutral $\mathrm{pH}$ [27], it is likely that the mutation does not sufficiently restrict the tunnel to completely exclude insertion of a fatty acid. In the crystal structure of the Bet3 A82V mutant, the protein indeed was found to be palmitoylated at cysteine 68, with an acyl chain occupying the hydrophobic tunnel (Kümmel/Heinemann, unpublished). We therefore engineered an additional mutation into Bet3 A82V. Alanine 138, which is located in the middle of the hydrophobic tunnel opposite to alanine 82 , was replaced by the bulky amino acid valine (Fig. 8A) to completely bock the tunnel. In accordance with this consideration, self-palmitoylation of Bet3 A82V-A138V is completely blocked at neutral pH (Fig. 8B).

Since lack of acylation increases the inherent instability of the Bet3 molecule [27], we compared the overall structure of Bet3 A82V-A138V with wild-type Bet3 using circular dichroism (CD) spectroscopy. However, the CD spectra for both molecules were similar, no indications for a random coil formation were detected (Fig. 8C), which indicates that the secondary structure of Bet3 was not changed significantly by the mutation.

Determination of the $\mathrm{K}_{\mathrm{D}}$ of the $\mathrm{A} 82 \mathrm{~V}-\mathrm{A} 138 \mathrm{~V}$ mutant for Pal-CoA revealed a reduction in its affinity to values of $15.8 \mu \mathrm{M}$, indicating that it still binds to Pal-CoA with only marginally reduced affinity (Fig. 6C and Table 3). From the fluorescence spectroscopy assay and the model we used to fit the data, the concentration of bound Pal-CoA and the number of acyl-CoA binding sites per Bet3 monomer was calculated (Table 3). Whereas wild-type Bet3, non-acylated Bet3 C68A and Bet3 A82V apparently contain three binding sites per Bet3 monomer, their number was found to be reduced to two in the Bet 3 mutant containing a completely blocked tunnel. This indicates that the hydrophobic tunnel is indeed a binding site for acyl-CoA, but that other sites with similar affinity exist as well, probably at the surface of the molecule. 


\section{Discussion}

In this study we have analysed binding of acyl-CoAs to Bet3 and investigated the chemical mechanism that allows transfer of the fatty acid from CoA to the acylation site of the protein, cysteine 68. The self-palmitoylating activity of wild-type Bet3 is enhanced by increasing the $\mathrm{pH}$ to slightly basic values (Fig. 1 and 2) suggesting that palmitoylation requires the formation of a deprotonated cysteine. At unphysiologically high $\mathrm{pH}$ values (9.5) even the Bet3 mutant containing alanine at the original acylation site is palmitoylated (Fig. 2), indicating that Bet3 has an inherent propensity to be acylated. One factor that controls S-acylation of Bet3 is the pKa value at which thiolate formation of individual putative acylation sites occurs. The authentic acylation site of Bet3, cysteine 68, is apparently in an environment that allows deprotonation at neutral $\mathrm{pH}$. Even a serine, that has a very high pKa value of 14 as free amino acid, is acylated at only slightly basic values ( $\mathrm{pH} 8.5$ ) when introduced at this position (Fig. 1 and 2). Thus, amino acids in the vicinity of the acylation site facilitate deprotonation of Cys 68 and even of Ser 68. A candidate for such an activity is the strictly conserved arginine 67, which is required for efficient acylation of Bet3 at neutral $\mathrm{pH}$ (Fig. 1). The functional defect of Bet3 R67E can be complemented by increasing the $\mathrm{pH}$ to 8.5. Furthermore, the crystal structure of Bet3 C68A shows that the guanidino group of Arg 67 has rotated towards the acylation site (Fig. 4) as compared to a previously published structure of wild-type Bet3 crystallized in the same space group [11]. The subtle structural change can be attributed to the fact that the palmitate ligand is negatively charged. This probably resembles charged reaction intermediates, in contrast to uncharged, covalently linked palmitate in the wild-type structure. These observations are also consistent with the assumption that Arg 67 stabilizes deprotonation of Cys 68. Alternatively, arginine 67 may polarize the carbonyl bond in the thioester linkage of Pal-CoA or stabilize the negative charge of a putative tetrahedral reaction intermediate.

The moderately conserved amino acids Asp 70 and Glu 73 apparently do not act as acceptor for protons released by thiolate formation of Cys 68. In contrast to Arg 67, their exchange did not diminish self-palmitoylation of Bet3 and did not cause a shift in the $\mathrm{pH}$ optimum of the reaction to basic values (Fig. 1 and 2). Instead, we observed increased acylation at acidic $\mathrm{pH}$, indicating that both amino acids might prevent acylation of Bet3 at unphysiologically low $\mathrm{pH}$ values. Thus, the inherent propensity of Bet 3 to be autoacylated is fine-tuned by the amino acids Asp 70, Glu 73 and especially by Arg 67 such that it occurs more rapidly (Fig. 3) and at neutral pH, and therefore at conditions which allow self-palmitoylation of Bet3 inside cells.

The second factor that controls efficient self-palmitoylation of Bet3 is enrichment of acyl-CoAs in the vicinity of putative acylation sites. The affinity of Bet3 for binding of Pal-CoA is relatively modest (micromolar, Fig. 6 and Table 2) compared to e.g. the N-myristoyl-transferase, which binds myristoyl-CoA with nanomolar affinity. However, the affinity of Bet3 for acyl-CoAs is approximately one order of magnitude higher compared to Pal-CoA concentrations inside cells $(5-150 \mu \mathrm{M})$ [40]. Bet3 binds the most common long chain acyl-CoAs (and also palmitoylation inhibitors with long carbon 
chains), but CoA alone or CoAs esterified to short carbon chains do not interact with Bet3 (Figs. 5-7). The binding properties of Bet 3 thus reflect the fatty acid composition of most S-acylated proteins, which contain a variety of long chain fatty acids [15]. Since wild-type Bet3 and Bet3 C68A have identical affinities for their lipid substrate (Table 2), stable binding of acyl-CoAs does not require fatty acid transfer to the acylation site.

Contrary to our initial assumption, Bet 3 mutants with a blocked tunnel bound to Pal-CoA with only slightly reduced affinity and revealed two acyl-CoA binding sites instead of three calculated for wildtype Bet3 (Table 3). This indicates that the hydrophobic tunnel is indeed a binding site for acyl-CoAs, but that other sites with similar affinity exist as well, probably at the surface of the molecule. Displaying the hydrophobic interaction potential of Bet3 revealed two continuous regions on its surface, which might interact with acyl chains (depicted green and blue in Fig. 8D).

Do these presumed acyl-CoA binding sites at the surface of the molecule play a role for selfpalmitoylation of Bet3 inside cells? Since the cytosolic acyl-CoA-binding protein (ACBP) binds its ligand with nanomolar affinity, virtually every cytosolic acyl-CoA molecule exists in a complex with ACBP [40]. Thus, free acyl-CoAs are available for Bet3 only at intracellular membranes, such as the ER and the Golgi apparatus, where they are needed for the synthesis of phospholipids and also for DHHC-catalyzed S-acylation of proteins. Bet3 has an intrinsic membrane binding activity, which targets the protein to the same intracellular location $[9,10]$. Bet3 probably does not penetrate deeply into the bilayer but resides at the membrane surface. If the interacting capacity of Bet 3 for acyl-CoAs were confined to the hydrophobic tunnel, it would be difficult for the molecule to interact with acylCoA's fatty acid, which is embedded in the hydrophobic interior of the bilayer. In contrast, a highaffinity acyl-CoA binding site on the surface of Bet3 might be more suitable to facilitate interaction with and extraction of acyl-CoAs from the membrane.

How is it conceivable that an acyl-CoA molecule bound to the surface of Bet 3 is inserted into the tunnel and its fatty acid transferred to cysteine 68 ? We have previously proposed a molecular model for the extrusion of the fatty acid from the hydrophobic tunnel of Bet3 [10]. The mechanism involves a rotation of approximately $30^{\circ}$ in the kinked helix $\alpha 2$ about a hinge region close to residue Gly 52 , coupled with a maximal displacement of approximately $9 \AA$ that would open the hydrophobic tunnel. Although purely speculative, it is possible that non-acylated Bet 3 contains an open tunnel. This state will be energetically highly unfavourable. Binding of acyl-CoAs to the surface of Bet 3 might trigger a conformational change that allows helix $\alpha 2$ to rotate back to its original position whereby the fatty acid of an acyl-CoA molecule would be inserted into the hydrophobic tunnel. Narrowing or blocking the tunnel by introduction of bulky amino acids reduces acylation of Bet3, probably because the conformational change that causes fatty acid insertion is hindered. The conformational change might be also sensitive to the $\mathrm{pH}$, since an extended hydrogen-bonding pattern seen in the Bet3 structure within the putative hinge region must be modulated to allow movement of helix $\alpha 2$. This assumption would explain how a partial restriction of the tunnel can be complemented by a slightly basic $\mathrm{pH}$ 
during the palmitoylation reaction. Insertion of acyl-CoA into the tunnel is a prerequisite for acylation, since Bet 3 containing a completely blocked tunnel is not acylated (Fig. 8).

As we already pointed out [10], there is some structural similarity between the palmitate-binding $\alpha$ helical face of Bet3 and the non-specific lipid transfer proteins (LTPs) from plants and mammals [4143]. LTPs bind a variety of lipids, including Pal-CoA, with dissociation constants in the micromolar range. A further similarity to Bet3 is their lipid-binding site, a hydrophobic tunnel extending through the protein and bending slightly in the middle. The hydrocarbon chain of each fatty acid is buried in the cavity, and the carboxyl group is localized at the aqueous interface in contact with surface residues and water molecules. In barley LTP, the binding pocket can contract or expand to accommodate fatty acids of various sizes. These conformational changes are accompanied by a bending motion in helix $\alpha \mathrm{A}$ and structural adjustments at the $\mathrm{C}$-terminus and at helix $\alpha \mathrm{C}$, which are similar to those proposed above for Bet3. It is thus conceivable that Bet3 is evolutionarily related to a lipid transfer protein. By mutational acquisition of a potential acylation site at the entrance of the tunnel and of amino acids that facilitate its deprotonation, Bet 3 has gained a self-palmitoylation activity that is used to stabilize the structure of the molecule. Other subunits of TRAPP exhibiting the same fold, such as TPC5/Trs31p and TPC6/Trs33p, do not contain an acylation site. Self-palmitoylation is lost, and the collapse of the molecule onto an empty cavity is prevented by bulky hydrophobic side chains that occupy the tunnel accommodating palmitate in Bet3.

In accordance with this assumption is our observation that the hydrophobic tunnel of the Trs33B:Bet3C68A dimer is occupied by non covalently bound palmitate (Fig. 4). Apparently, Bet3, which can not stabilize its structure by self-palmitoylation of cysteine 68 , nevertheless inserts a fatty acid into the tunnel. Non covalently bound palmitate might be also present in purified wild-type Bet3, but this moiety can be exchanged by Pal-CoA, for which Bet3 has a much higher affinity, in order for selfpalmitoylation to occur.

In summary, our results suggest the following mechanism mediating self-palmitoylation of Bet3. Acyl-CoA binds to the surface of the molecule, and its fatty acid is subsequently inserted into the hydrophobic tunnel. The thioester bond of the Pal-CoA molecule is now situated at the entrance of the tunnel where the fatty acid acceptor, cysteine 68 , is located. Facilitated by amino acids in its vicinity, especially by arginine 67, cysteine 68 is deprotonated. The resulting thiolate functions as a nucleophile attacking the carbonyl-C of the C-S bond in Pal-CoA. The fatty acid is then attached to cysteine 68, and CoA is released from Bet3. Since an acylated Bet 3 molecule is no longer available for a second round of catalysis, this mechanism is different from a classical enzymatic reaction. However, the speed of the reaction in vitro, its $\mathrm{pH}$ and $\mathrm{Pal}-\mathrm{CoA}$ requirements and the observation that Bet 3 does not require functional DHHC proteins for its acylation inside cells [28], distinguishes self-palmitoylation of Bet3 from purely chemical acylation reactions described previously [19-25]. 


\section{Achnowledgment}

This work was funded by the DFG (SFB 740, TP C1) and supported by the Fonds der Chemischen Industrie. We thank Ingrid Poese for experimental assistance and the staff at ESRF BM14 for excellent beamline support. 


\section{References}

1. Barrowman J, Sacher M, Ferro-Novick S (2000) TRAPP stably associates with the Golgi and is required for vesicle docking. Embo J 19: 862-869

2. Sacher M, Barrowman J, Wang W, Horecka J, Zhang Y, Pypaert M, Ferro-Novick S (2001) TRAPP I implicated in the specificity of tethering in ER-to-Golgi transport. Mol Cell 7: 433-442

3. Sacher M, Jiang Y, Barrowman J, Scarpa A, Burston J, Zhang L, Schieltz D, Yates JR, 3rd, Abeliovich H, Ferro-Novick S (1998) TRAPP, a highly conserved novel complex on the cisGolgi that mediates vesicle docking and fusion. Embo J 17: 2494-2503

4. Kummel D, Heinemann U (2008) Diversity in structure and function of tethering complexes: evidence for different mechanisms in vesicular transport regulation. Curr Protein Pept Sci 9: 197-209

5. Sacher M, Kim YG, Lavie A, Oh BH, Segev N (2008) The TRAPP complex: insights into its architecture and function. Traffic 9: 2032-2042

6. Cai Y, Chin HF, Lazarova D, Menon S, Fu C, Cai H, Sclafani A, Rodgers DW, De La Cruz EM, Ferro-Novick S, Reinisch KM (2008) The structural basis for activation of the Rab Ypt1p by the TRAPP membrane-tethering complexes. Cell 133: 1202-1213

7. Jones S, Newman C, Liu F, Segev N (2000) The TRAPP complex is a nucleotide exchanger for Ypt1 and Ypt31/32. Mol Biol Cell 11: 4403-4411

8. Wang W, Sacher M, Ferro-Novick S (2000) TRAPP stimulates guanine nucleotide exchange on Ypt1p. J Cell Biol 151: 289-296

9. Kim YG, Sohn EJ, Seo J, Lee KJ, Lee HS, Hwang I, Whiteway M, Sacher M, Oh BH (2005) Crystal structure of bet 3 reveals a novel mechanism for Golgi localization of tethering factor TRAPP. Nat Struct Mol Biol 12: 38-45

10. Turnbull AP, Kummel D, Prinz B, Holz C, Schultchen J, Lang C, Niesen FH, Hofmann KP, Delbruck H, Behlke J, Muller EC, Jarosch E, Sommer T, Heinemann U (2005) Structure of palmitoylated BET3: insights into TRAPP complex assembly and membrane localization. Embo J 24: 875-884

11. Kim MS, Yi MJ, Lee KH, Wagner J, Munger C, Kim YG, Whiteway M, Cygler M, Oh BH, Sacher M (2005) Biochemical and crystallographic studies reveal a specific interaction between TRAPP subunits Trs33p and Bet3p. Traffic 6: 1183-1195

12. Kim YG, Raunser S, Munger C, Wagner J, Song YL, Cygler M, Walz T, Oh BH, Sacher M (2006) The architecture of the multisubunit TRAPP I complex suggests a model for vesicle tethering. Cell 127: 817-830

13. Kummel D, Muller JJ, Roske Y, Henke N, Heinemann U (2006) Structure of the Bet3-Tpc6B core of TRAPP: two Tpc6 paralogs form trimeric complexes with Bet3 and Mum2. J Mol Biol 361: $22-32$ 
14. Kummel D, Muller JJ, Roske Y, Misselwitz R, Bussow K, Heinemann U (2005) The structure of the TRAPP subunit TPC6 suggests a model for a TRAPP subcomplex. EMBO Rep 6: 787793

15. Linder ME, Deschenes RJ (2007) Palmitoylation: policing protein stability and traffic. Nat Rev Mol Cell Biol 8: 74-84

16. Pfanner N, Glick BS, Arden SR, Rothman JE (1990) Fatty acylation promotes fusion of transport vesicles with Golgi cisternae. J Cell Biol 110: 955-961

17. Pfanner N, Orci L, Glick BS, Amherdt M, Arden SR, Malhotra V, Rothman JE (1989) Fatty acyl-coenzyme A is required for budding of transport vesicles from Golgi cisternae. Cell 59: 95102

18. Quesnel S, Silvius JR (1994) Cysteine-containing peptide sequences exhibit facile uncatalyzed transacylation and acyl-CoA-dependent acylation at the lipid bilayer interface. Biochemistry 33 : 13340-13348

19. Belanger C, Ansanay H, Qanbar R, Bouvier M (2001) Primary sequence requirements for Sacylation of beta(2)-adrenergic receptor peptides. FEBS Lett 499: 59-64

20. Bizzozero OA, Bixler HA, Pastuszyn A (2001) Structural determinants influencing the reaction of cysteine-containing peptides with palmitoyl-coenzyme A and other thioesters. Biochim Biophys Acta 1545: 278-288

21. Duncan JA, Gilman AG (1996) Autoacylation of G protein alpha subunits. J Biol Chem 271: 23594-23600

22. Veit M (2000) Palmitoylation of the 25-kDa synaptosomal protein (SNAP-25) in vitro occurs in the absence of an enzyme, but is stimulated by binding to syntaxin. Biochem J 345 Pt 1: 145151

23. Veit M, Sachs K, Heckelmann M, Maretzki D, Hofmann KP, Schmidt MF (1998) Palmitoylation of rhodopsin with S-protein acyltransferase: enzyme catalyzed reaction versus autocatalytic acylation. Biochim Biophys Acta 1394: 90-98

24. Bano MC, Jackson CS, Magee AI (1998) Pseudo-enzymatic S-acylation of a myristoylated yes protein tyrosine kinase peptide in vitro may reflect non-enzymatic S-acylation in vivo. Biochem J 330 ( Pt 2): 723-731

25. Bizzozero OA, McGarry JF, Lees MB (1987) Autoacylation of myelin proteolipid protein with acyl coenzyme A. J Biol Chem 262: 13550-13557

26. Tsutsumi R, Fukata Y, Fukata M (2008) Discovery of protein-palmitoylating enzymes. Pflugers Arch 456: 1199-1206

27. Kummel D, Heinemann U, Veit M (2006) Unique self-palmitoylation activity of the transport protein particle component Bet3: a mechanism required for protein stability. Proc Natl Acad Sci U S A 103: 12701-12706 
28. Roth AF, Wan J, Bailey AO, Sun B, Kuchar JA, Green WN, Phinney BS, Yates JR, 3rd, Davis NG (2006) Global analysis of protein palmitoylation in yeast. Cell 125: 1003-1013

29. Bhatnagar RS, Futterer K, Waksman G, Gordon JI (1999) The structure of myristoylCoA:protein N-myristoyltransferase. Biochim Biophys Acta 1441: 162-172

30. Wang W, Malcolm BA (1999) Two-stage PCR protocol allowing introduction of multiple mutations, deletions and insertions using QuikChange Site-Directed Mutagenesis. Biotechniques 26: $680-682$

31. Heinemann U, Bussow K, Mueller U, Umbach P (2003) Facilities and methods for the highthroughput crystal structural analysis of human proteins. Acc Chem Res 36: 157-163

32. McCoy AJ, Grosse-Kunstleve RW, Adams PD, Winn MD, Storoni LC, Read RJ (2007) Phaser crystallographic software. J Appl Crystallogr 40: 658-674

33. Emsley P, Cowtan K (2004) Coot: model-building tools for molecular graphics. Acta Crystallogr D Biol Crystallogr 60: 2126-2132

34. Murshudov GN, Vagin AA, Dodson EJ (1997) Refinement of macromolecular structures by the maximum-likelihood method. Acta Crystallogr D Biol Crystallogr 53: 240-255

35. Heck M, Hofmann KP (1993) G-protein-effector coupling: a real-time light-scattering assay for transducin-phosphodiesterase interaction. Biochemistry 32: 8220-8227

36. Dietrich LE, Ungermann C (2004) On the mechanism of protein palmitoylation. EMBO Rep 5: 1053-1057

37. Pace CN, Grimsley GR, Scholtz JM (2009) Protein ionizable groups: pK values and their contribution to protein stability and solubility. J Biol Chem 284: 13285-13289

38. Resh MD (2006) Use of analogs and inhibitors to study the functional significance of protein palmitoylation. Methods 40: 191-197

39. Roth AF, Feng Y, Chen L, Davis NG (2002) The yeast DHHC cysteine-rich domain protein Akr1p is a palmitoyl transferase. J Cell Biol 159: 23-28

40. Faergeman NJ, Knudsen J (1997) Role of long-chain fatty acyl-CoA esters in the regulation of metabolism and in cell signalling. Biochem J 323 ( Pt 1): 1-12

41. Hamilton JA (2004) Fatty acid interactions with proteins: what X-ray crystal and NMR solution structures tell us. Prog Lipid Res 43: 177-199

42. Lerche MH, Kragelund BB, Bech LM, Poulsen FM (1997) Barley lipid-transfer protein complexed with palmitoyl CoA: the structure reveals a hydrophobic binding site that can expand to fit both large and small lipid-like ligands. Structure 5: 291-306

43. Lerche MH, Poulsen FM (1998) Solution structure of barley lipid transfer protein complexed with palmitate. Two different binding modes of palmitate in the homologous maize and barley nonspecific lipid transfer proteins. Protein Sci 7: 2490-2498 


\section{Figure legends}

\section{Figure 1: Testing the $\mathrm{pH}$ dependence of palmitoylation of Bet3}

A: Orthogonal views of the crystal structure of human Bet3: The covalently bound fatty acid inserted into the hydrophobic tunnel, amino acids mutated in this study and the single tryptophan (W 96) used for fluorescence spectroscopy are highlighted.

B and C: Comparison of the self-palmitoylating activity of Bet3 mutants relative to wild-type Bet3 at different $\mathrm{pH}$ values.

B: Equal amounts of purified wild-type GST-Bet3 (WT) and GST-Bet3 mutants containing aminoacid substitutions at cysteine 68 (C68S, C68A) or in the vicinity of the palmitoylation site (R67E) were incubated with $\left[{ }^{3} \mathrm{H}\right]-\mathrm{Pal}-\mathrm{CoA}$ in PIPES/ $\mathrm{NaCl}$ at $\mathrm{pH} 6.5$ or Tris/ $\mathrm{NaCl}$ buffer at $\mathrm{pH} 7.5$ or 8.5 for $20 \mathrm{~min}$ at $30^{\circ} \mathrm{C}$. Samples were then subjected to SDS-PAGE and fluorography. Shown are the resulting fluorograms and the quantification of individual bands $(\mathrm{wt}=100 \%)$ by densitometry. C: Similar amounts of purified wild-type GST-Bet3 and GST-Bet3 mutants containing amino-acid substitutions at aspartate $70(\mathrm{D} 70 \mathrm{~N})$ and glutamate 73 (E73Q) were incubated with Pal-CoA as described in B. Shown are the resulting fluorograms and the quantification of individual bands (D67E $=100 \%)$ by densitometry.

Figure 2: Comparison of the self-palmitoylating activity of Bet3 mutants at different pH values. A: Wild-type GST-Bet3 (WT) and R67E, C68S, C68A mutants were incubated with [ $\left.{ }^{3} \mathrm{H}\right]-\mathrm{Pal}-\mathrm{CoA}$ in PIPES/NaCl buffer at $\mathrm{pH} 5.5,6.5$ or 7.5 or in Tris/ $\mathrm{NaCl}$ buffer at $\mathrm{pH} 7.5,8.5$ or 9.5 for $20 \mathrm{~min}$ at $30^{\circ} \mathrm{C}$.

B: Comparison of the self-palmitoylating activity of wild-type GST-Bet3 and GST-Bet3 E73Q, D70N, and the double mutant E73Q-D70N at different $\mathrm{pH}$ values as described in Figure 2A. The fluorograms were quantified by densitometry of bands, and relative palmitoylation (absorbance of bands at $\mathrm{pH} 9.5$ $=100 \%)$ is plotted against $\mathrm{pH}$.

Note that the strength of the self-palmitoylation reaction (intensity of bands) cannot be compared between wild-type and mutant Bet3 proteins, since individual fluorograms were exposed for different time periods.

Figure 3: Self-palmitoylation of Bet3 is retarded in mutants which are less efficiently palmitoylated at neutral $\mathrm{pH}$

Purified GST-Bet3 proteins, wild-type and mutants as described in Fig. 1, were incubated at $\mathrm{pH} 7.5$ with $\left[{ }^{3} \mathrm{H}\right]$-Pal-CoA for $1,2.5,5,7.5,10,15$ or 30 minutes at $30{ }^{\circ} \mathrm{C}$ prior to SDS-PAGE and fluorography. The fluorograms were quantified by densitometry of bands, and relative palmitoylation (absorbance of bands after $30 \mathrm{~min}$ of incubation $=100 \%$ ) is plotted against the time of incubation. Note that individual fluorograms were exposed for different time periods, and, thus, intensity of bands cannot be compared between wild-type and mutant Bet3 proteins. 


\section{Figure 4: X-ray structural analysis of the Trs33B:Bet3C68A mutant}

A: The hydrophobic tunnel of Bet3 C68A is occupied by an elongated molecule. A prominent, unaccounted electron density is visible after building the peptide chain of Bet3 (upper panel: $2 F_{\mathrm{o}}-F_{\mathrm{c}}$ map, blue at $0.9 \sigma$ contour level and $F_{\mathrm{o}}-F_{\mathrm{c}}$ map, green at $3 \sigma$ contour level). Palmitate could be modelled into the hydrophobic channel (lower panel: $2 F_{\mathrm{o}}-F_{\mathrm{c}}$ map, blue at $1.0 \sigma$ contour level). B: Superposition of the $\alpha 2-\alpha 3$ loop region of mutant Bet3 C68A (green, complex presented here) and wild-type Bet3 (cyan, in the Trs33A:Bet3 complex , PDB: 2C0J) which were crystallized in the same space group.

\section{Figure 5: Long chain acyl-CoAs inhibit self-palmitoylation of Bet3 at micromolar concentration} A: Myristoyl-CoA, palmitoyl-CoA, stearoyl-CoA and 9-cis-oleoyl-CoA at the indicated final concentrations were incubated with wild-type GST-Bet 3 and $\left[{ }^{3} \mathrm{H}\right]-\mathrm{Pal}-\mathrm{CoA}$ for $15 \mathrm{~min}$ at $30{ }^{\circ} \mathrm{C}$. The resulting fluorograms are shown.

B: Acetyl-CoA and octanoyl-CoA at the indicated final concentrations were incubated with wild-type GST-Bet 3 and $\left[{ }^{3} \mathrm{H}\right]-\mathrm{Pal}-\mathrm{CoA}$ for $15 \mathrm{~min}$ at $30{ }^{\circ} \mathrm{C}$. Note that millimolar concentrations of these CoA derivatives were required to reveal a slight reduction in palmitoylation.

C: Myr-X-CoA (a non-hydrolysable Myr-CoA analog with a thioether instead of a thioester linking myristate and $\mathrm{CoA}$ ) at the indicated final concentration was incubated with wild-type GST-Bet 3 and $\left[{ }^{3} \mathrm{H}\right]-\mathrm{Pal}-\mathrm{CoA}$ for $15 \mathrm{~min}$ at $30^{\circ} \mathrm{C}$.

\section{Figure 6: Fluorescence spectroscopy to analyse binding of Acyl-CoAs to Bet3}

A: Left panel: Emission spectra of purified wild-type Bet3 between $310 \mathrm{~nm}$ and $410 \mathrm{~nm}$. Excitation at $295 \mathrm{~nm}$. The uppermost trace represents the intrinsic tryptophan fluorescence of Bet3 in the absence of acyl-CoA. It is successively quenched upon addition of palmitoyl-CoA which was added in steps of 10 $\mu \mathrm{M}$ (final concentration) to Bet3 (subsequent traces). No further reduction of the tryptophan fluorescence occurs at a final Pal-CoA concentration of $75 \mu \mathrm{M}$ (undermost traces). Right panel: Quantitative analysis of binding of Pal-CoA to wild-type Bet3 (described in C). Error bars indicate the standard deviation calculated from three experiments.

B: Left panel: Emission spectra of purified Bet3: Similar to A, but acetyl-CoA was added to Bet3 in steps of $1 \mathrm{mM}$ (final concentration). Note that the intrinsic tryptophan fluorescence is not completely quenched, even at a final concentration of $10 \mathrm{mM}$ acetyl-CoA. Right panel: Quantitative analysis of acetyl-CoA binding to wild-type Bet3 (described in C). Error bars indicate the standard deviation calculated from three experiments.

C: Quantitative analysis of binding of Pal-CoA to wild-type and mutant Bet3. Fitting of fluorescence intensities at the emission maximum $(344 \mathrm{~nm})$ were normalized $\left(\left(\mathrm{F}-\mathrm{F}_{0}\right) / \mathrm{F}_{0}, \mathrm{~F}_{0}\right.$ : fluorescence intensity without ligand) and plotted against the Pal-CoA concentration. The data points were fitted and the number of acyl-CoA bindings sites listed in table 2 and 3 were calculated as described in materials and 
methods. Error bars indicate the standard deviation calculated from three experiments. Where not indicated error bars are not larger than the symbols.

Figure 7: Testing inhibitors of the self-palmitoylation reaction

A: Palmitate or 2-bromo-palmitate at the indicated final concentrations were incubated with wild-type GST-Bet 3 and $\left[{ }^{3} \mathrm{H}\right]-\mathrm{Pal}-\mathrm{CoA}$ for $15 \mathrm{~min}$ at $30^{\circ} \mathrm{C}$.

B: Lyso-phosphatidylcholine, cerulenin or tunicamycin at the indicated final concentrations were incubated with wild-type GST-Bet 3 and $\left[{ }^{3} \mathrm{H}\right]-\mathrm{Pal}-\mathrm{CoA}$ for 15 min at $30{ }^{\circ} \mathrm{C}$. \#: $1 \%$ chloroform, the solvent for the three inhibitors, was added to the sample.

C: The designated nucleotides at the indicated final concentrations and $\mathrm{MgCl}_{2}(1 \mathrm{mM})$ were incubated with wild-type GST-Bet 3 and $\left[{ }^{3} \mathrm{H}\right]-\mathrm{Pal}-\mathrm{CoA}$ for $15 \mathrm{~min}$ at $30^{\circ} \mathrm{C}$.

\section{Figure 8: Testing a Bet3 mutant with a completely blocked hydrophobic tunnel}

A: Detail of the Bet 3 crystal structure showing the hydrophobic tunnel. The fatty acid is depicted as yellow space-filling model. The amino acids mutated in A82V-A138V to block the hydrophobic tunnel are modelled purple with their electron densities indicated. The tunnel is narrowed by the two opposing valines (V138 top, V82 bottom).

B: CD spectra of wild-type Bet3 and Bet3 A82V-A138V indicate that the secondary structure of Bet3 was not changed significantly by the mutation.

C: Similar amounts of purified wild-type GST-Bet3 and GST-Bet3 A82V-A138V were incubated with $\left[{ }^{3} \mathrm{H}\right]-\mathrm{Pal}-\mathrm{CoA}$ in PIPES/NaCl at $\mathrm{pH} 6.5$ or Tris/ $\mathrm{NaCl}$ buffer at $\mathrm{pH} 7.5$ or 8.5 for $20 \mathrm{~min}$ at $30^{\circ} \mathrm{C}$.

D: Patches of hydrophobic amino acids at the surface of Bet3 that might bind the fatty acid of AcylCoAs. The green area contains the amino acids L60, A61, V65, G66, V77, V81, L87, G88, I89, I106, L107, P111, G159, V160, the blue area consists of F71, I93, W96, P98, A99, G100, A145, F167, I168, I171. 


\section{Table 1}

Data collection and refinement statistics for the structure of the Trs33B:Bet3 C68A complex.

Values in parentheses refer to the outer shell of reflections.

\begin{tabular}{|c|c|}
\hline \multicolumn{2}{|l|}{ X-ray diffraction data } \\
\hline Space group & $\mathrm{P} 2_{1} 2_{1} 2_{1}$ \\
\hline Unit cell a $[\AA]$ & 47.17 \\
\hline $\mathrm{b}[\AA]$ & 69.26 \\
\hline $\mathrm{c}[\AA]$ & 121.38 \\
\hline Resolution $[\AA]$ & $30-2.0 \quad(2.1-2.0)$ \\
\hline Unique reflections & $26752(3311)$ \\
\hline Redundancy & $4.05 \quad(4.15)$ \\
\hline$<\mathrm{I} / \sigma(\mathrm{I})>$ & $16.8 \quad(3.0)$ \\
\hline Completeness [\%] & $94.9 \quad(87.6)$ \\
\hline $\mathrm{R}(\operatorname{sym})[\%]$ & $6.3(50.1)$ \\
\hline \multicolumn{2}{|l|}{ Refinement } \\
\hline Resolution $[\AA]$ & $25-2.0$ \\
\hline $\mathrm{R}$ (work) / R(free) [\%] & $19.7 / 23.4$ \\
\hline RMSD bond distances $[\AA]$ & 0.009 \\
\hline RMSD bond angles $\left[{ }^{\circ}\right]$ & 1.117 \\
\hline Mean $B$ value $\left[\AA^{2}\right]$ protein & 42.2 \\
\hline water $(100)$ & 41.5 \\
\hline palmitate (1) & 56.4 \\
\hline \multicolumn{2}{|l|}{ Ramachandran statistics } \\
\hline Residues in most favored regions [\%] & 97.3 \\
\hline Residues in additionally allowed regions [\%] & 2.7 \\
\hline
\end{tabular}




\section{Table 2}

$\mathrm{K}_{\mathrm{D}}[\mu \mathrm{M}]$ values including standard deviation for binding of various acyl-CoAs to wild-type Bet3 and to the non-acylated C68A mutant as calculated from at least three different fluorescence spectroscopy measurements..

\begin{tabular}{|lcc|}
\hline Acyl-CoA & Bet3 wt & Bet3 C68A \\
\hline Stearoyl-CoA & $1.3 \pm 0.8$ & $2.9 \pm 0.8$ \\
Oleoyl-CoA & $2.1 \pm 0.6$ & $1.1 \pm 0.3$ \\
Palmitoyl-CoA & $1.8 \pm 0.7$ & $1.6 \pm 0.6$ \\
Myristoyl-CoA & $2.0 \pm 0.8$ & $2.3 \pm 1.6$ \\
Octanoyl-CoA & $7252 \pm 1412$ & $11256 \pm 3055$ \\
Acetyl-CoA & $7624 \pm 2110$ & $7321 \pm 1327$ \\
2-Br-Palmitate & $1.3 \pm 0.5$ & $1.9 \pm 0.3$ \\
\hline
\end{tabular}




\section{Table 3}

$\mathrm{K}_{\mathrm{D}}$ values $[\mu \mathrm{M}]$ including standard deviation and number (\#) of interaction sites for binding of Pal-CoA to wild-type and mutant Bet3 as calculated from at least three different fluorescence spectroscopy measurements.

\begin{tabular}{|lcc|}
\hline Bet3 mutant & $\mathrm{K}_{\mathrm{D}}$ & \# of interaction sites \\
\hline wild type & $1.8 \pm 0.7$ & $3.5 \pm 0.2$ \\
C68A & $1.6 \pm 0.6$ & $3.3 \pm 0.2$ \\
A82V & $4.0 \pm 0.5$ & $3.7 \pm 0.1$ \\
A82V-A138V & $15.8 \pm 2.3$ & $2.1 \pm 0.4$ \\
\hline
\end{tabular}


A
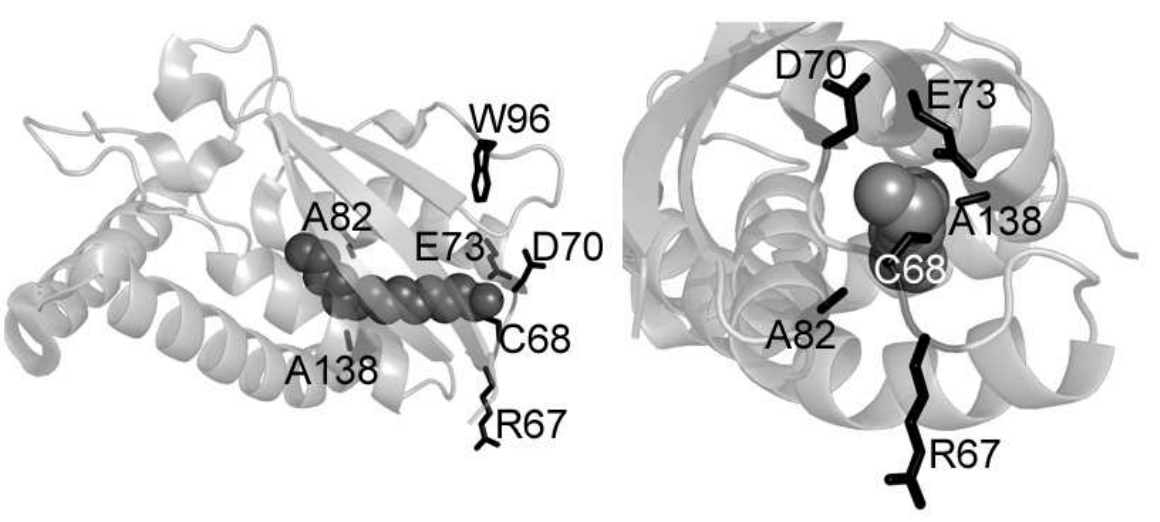

B

$\mathrm{pH} 6.5$

$\mathrm{pH} 7.5$

WT C68A C68SR67E WT C68A C68S R67E WT C68A C68S R67E
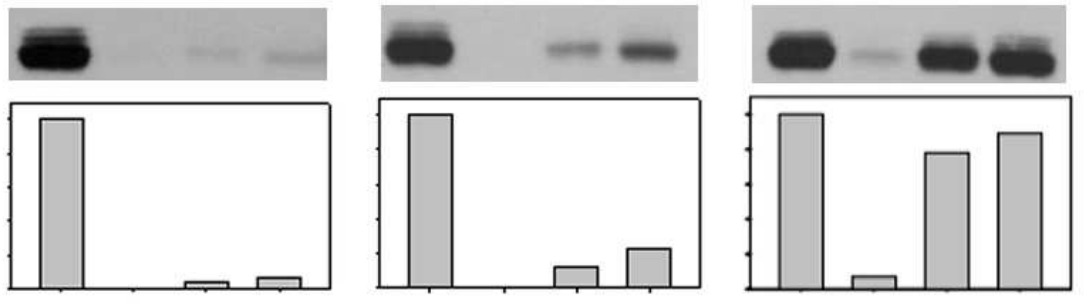

C
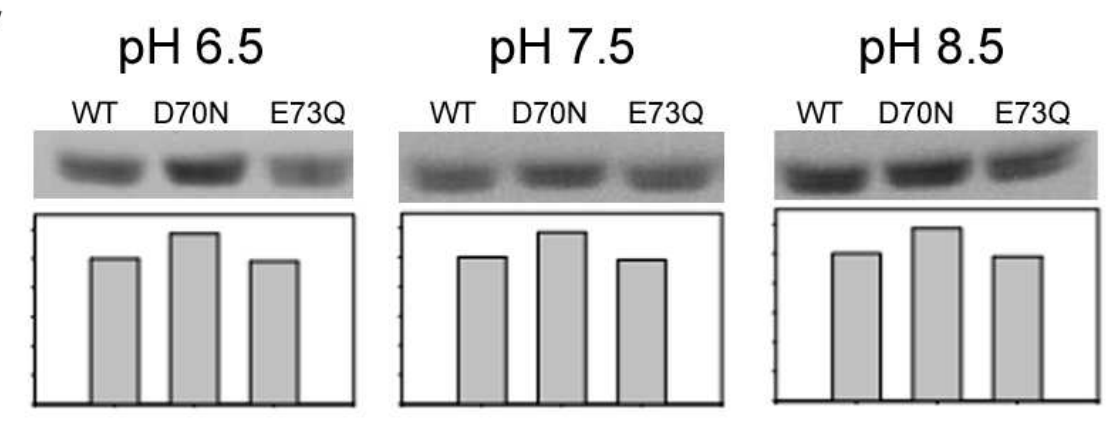


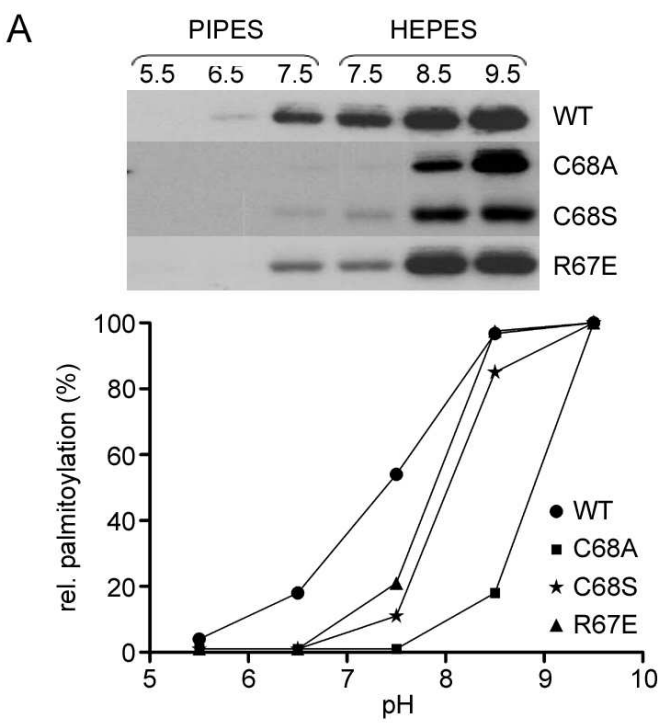

B
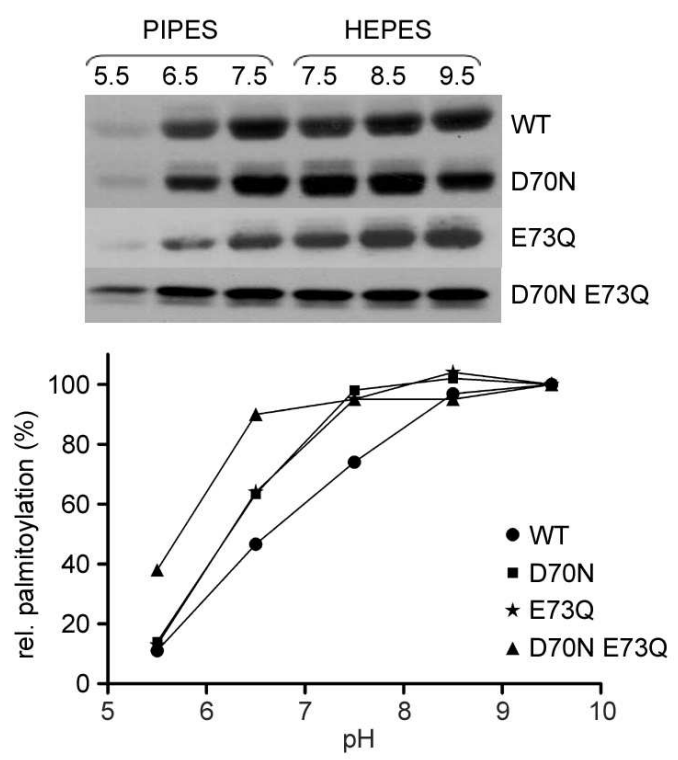
A

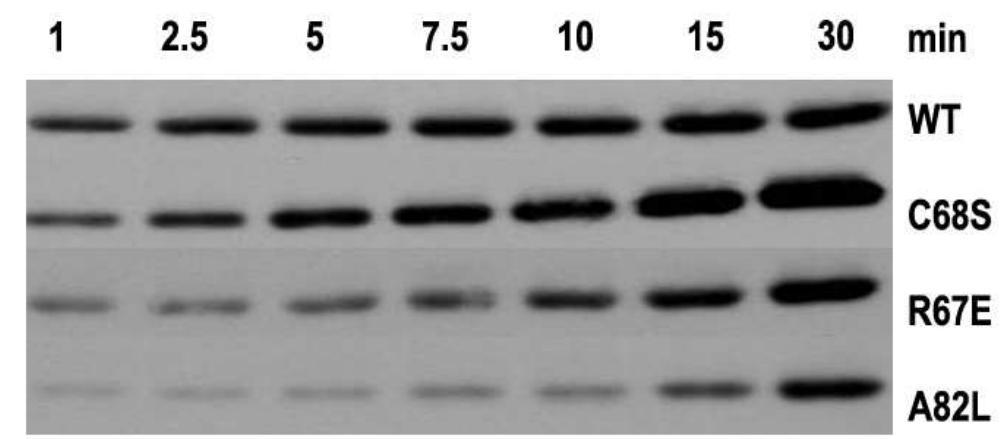

B

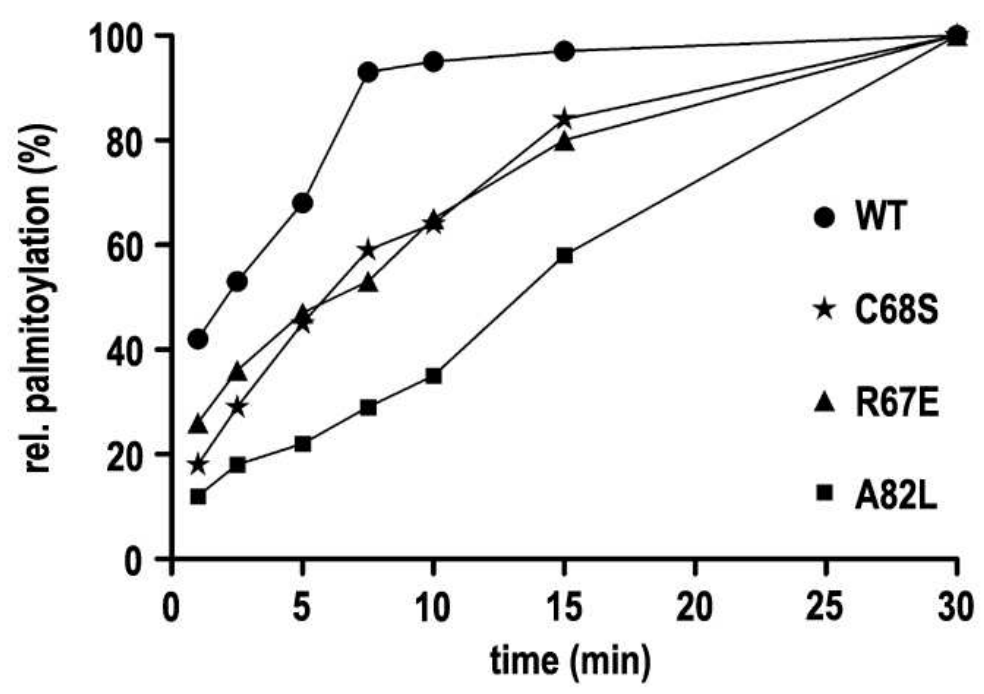

41

42

43

44

45

46

47

48

49

50

51

52

53

54

55

56

57

58

59

60 


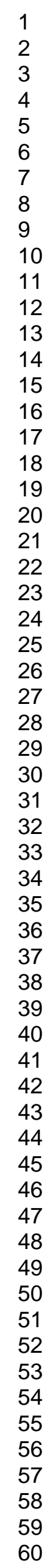

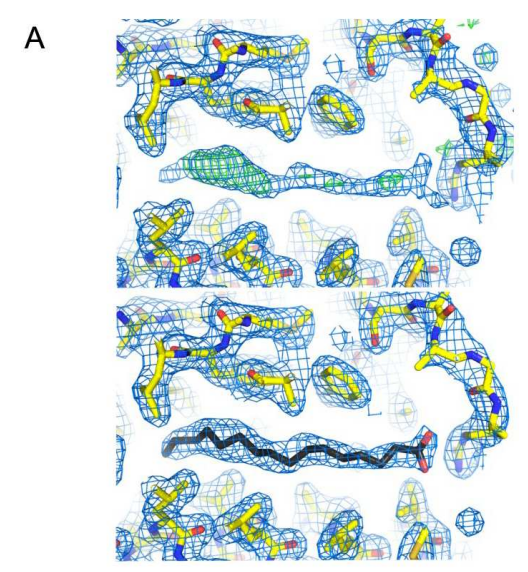

B

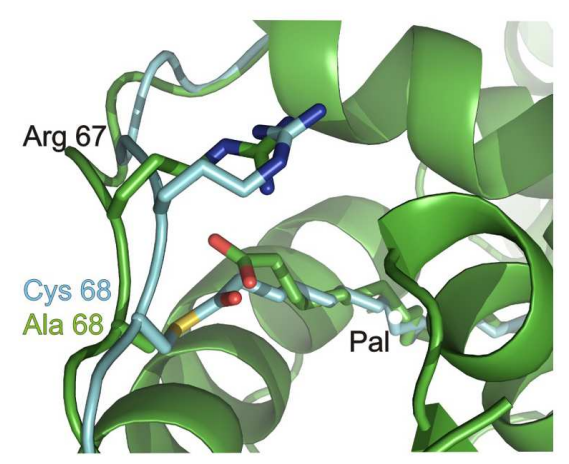




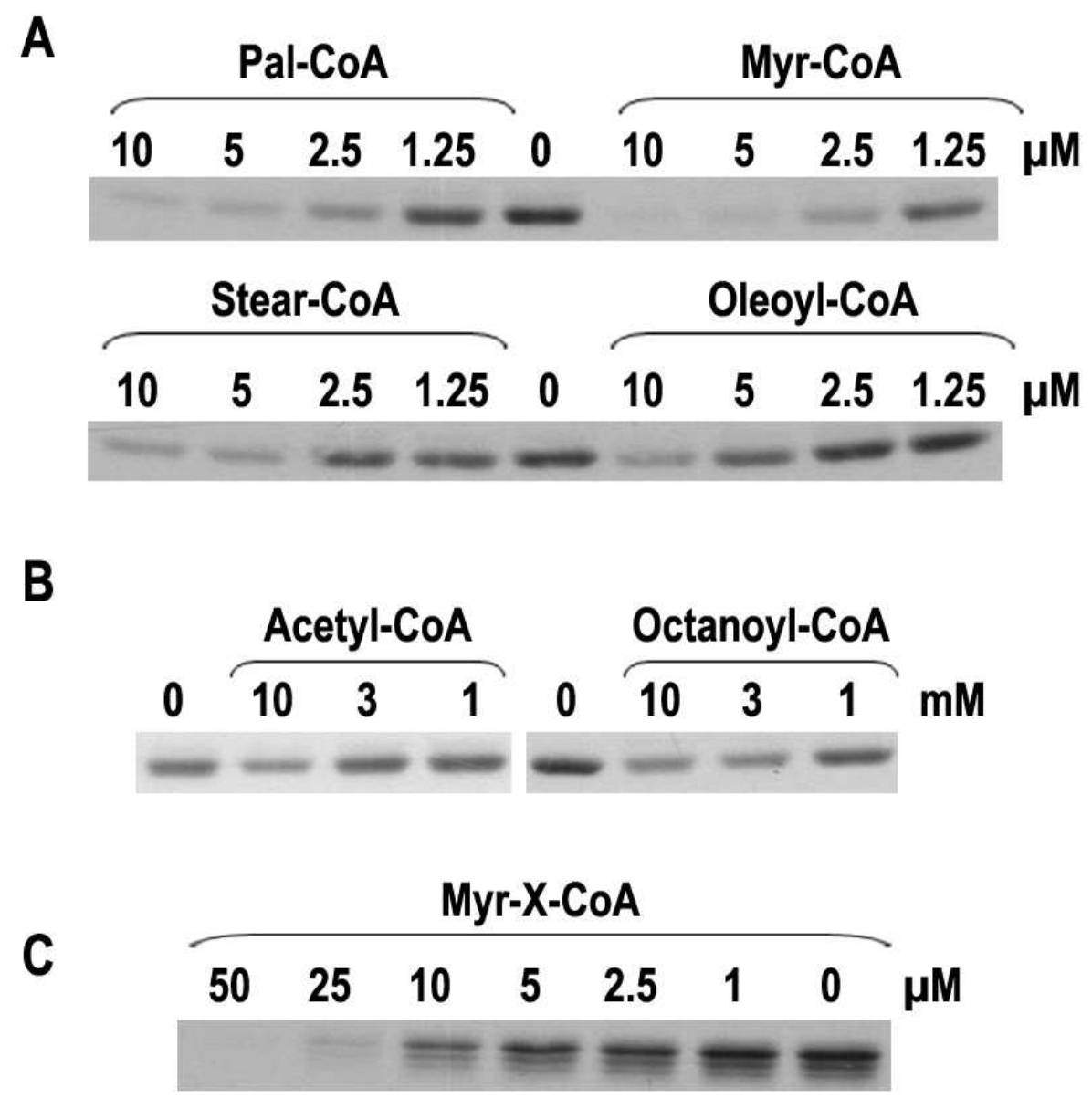




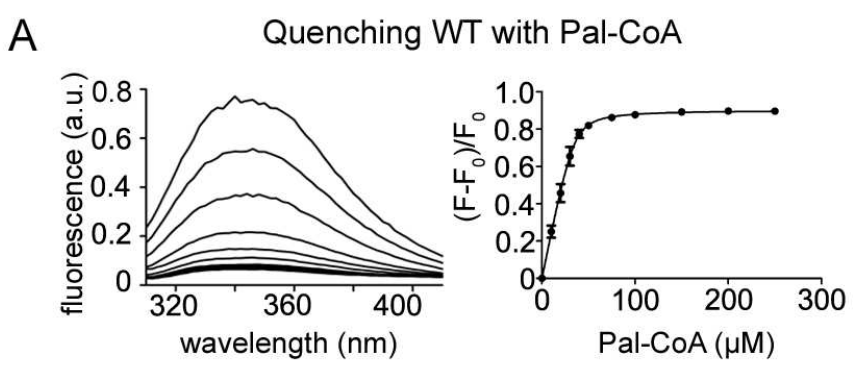

B

Quenching WT with Ac-CoA
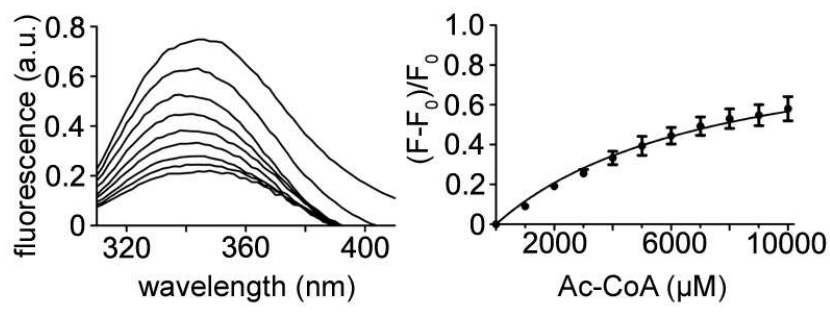

C

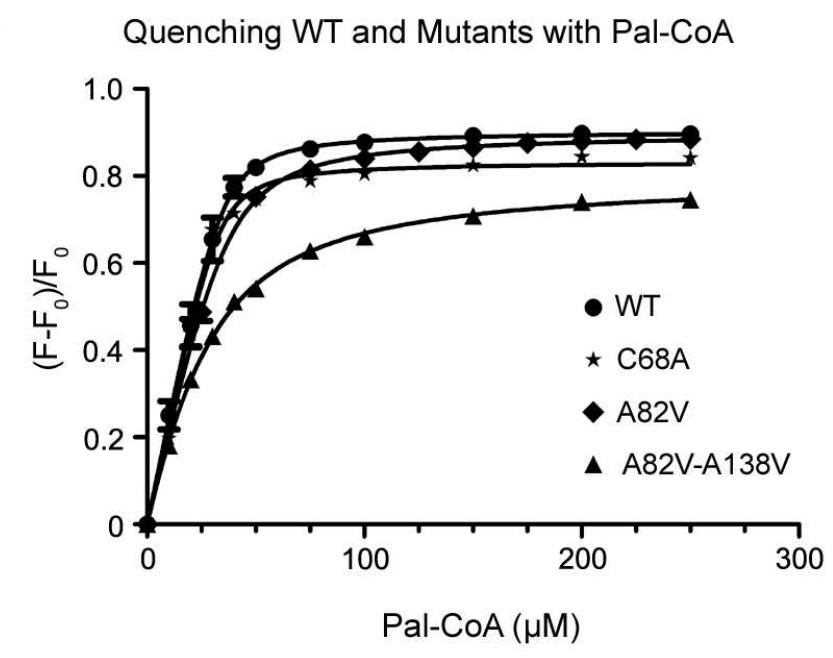


A

\begin{tabular}{|c|c|c|c|c|c|}
\hline Palmitat & & & \multicolumn{3}{|c|}{ Br-palmitate } \\
\hline 100010010 & 1 & 0 & 1000100 & 10 & 1 \\
\hline 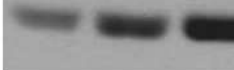 & & & & & $=$ \\
\hline
\end{tabular}

B

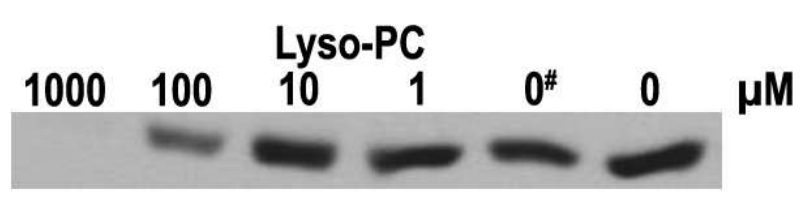

Tunicamycin

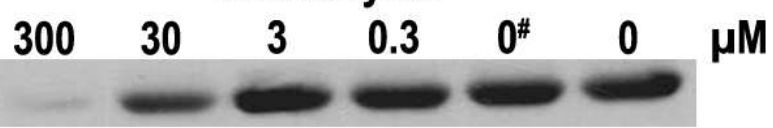

$1000 \quad 100 \quad \begin{array}{lllll}\text { Cerulenin } \\ 10 & 1 & 0^{\#} & 0 & \mu M\end{array}$

C

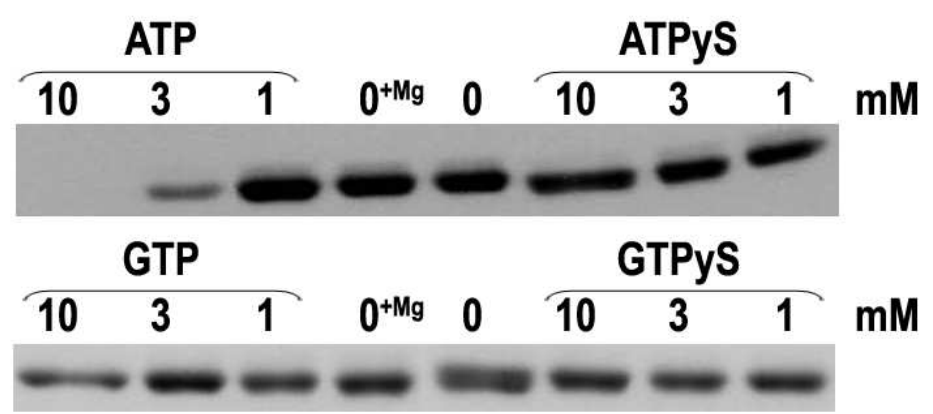


A

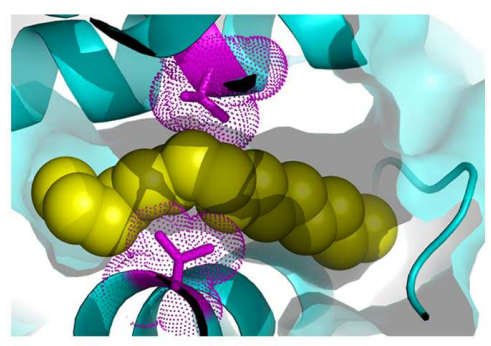

C

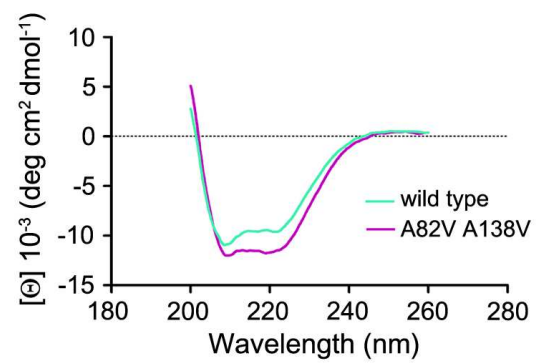

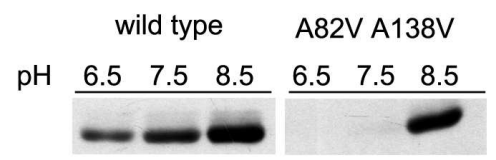

D

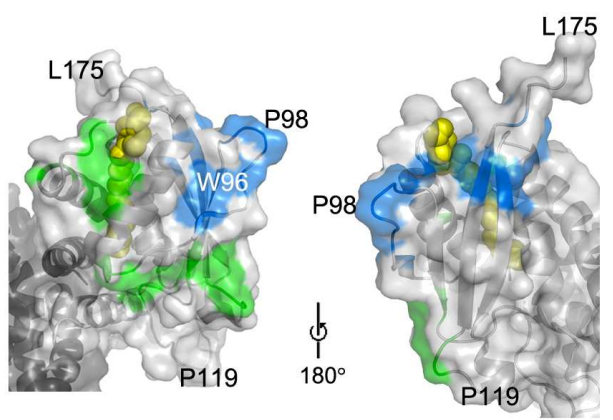

